Check for updates

Cite this: RSC Adv., 2018, 8, 14740

Received 2nd February 2018

Accepted 4th April 2018

DOI: $10.1039 / \mathrm{c} 8 \mathrm{ra01044g}$

rsc.li/rsc-advances

\section{Fabrication and characterization of antistatic epoxy composite with multi-walled carbon nanotube- functionalized melamine foam}

\author{
Xueliang Feng, Jiangang Wang, Chen Zhang, Zhongjie Du, ${ }^{*}$ Hangquan Li \\ and Wei Zou (iD *
}

A novel strategy for synthesizing an antistatic epoxy composite was carried out. Pre-embedded antistatic melamine foam was first synthesized and then used to prepare an antistatic epoxy composite. Azidized polyacrylic acid (APAA) was grafted onto multiwalled carbon nanotubes (APAA-MWCNTs) by direct functional modification of the MWCNT sidewalls. Melamine was then covalently bonded to MWCNTs (MAAPAA-MWCNTs). The chemical structure of MA-APAA-MWCNTs was characterized by FT-IR spectroscopy, EDS, TGA, Raman spectroscopy, and TEM. As an antistatic agent, MA-APAA-MWCNTs utilized the functional groups of the surface to participate in the formation of melamine foam by reaction with paraformaldehyde. The surface resistivity was decreased to $3.6 \times 10^{8} \Omega \mathrm{sq}^{-1}$ when the loading of MWCNTs was $2.4 \mathrm{~kg} \mathrm{~m}^{-3}$. The prepared antistatic foam at different compression ratios was immersed in epoxy resin, which was then cured. When the compression ratio reached $40 \%$, the surface resistivity and volume resistivity, respectively, reached $1.05 \times 10^{8} \Omega \mathrm{sq}^{-1}$ and $3.5 \times 10^{8} \Omega \mathrm{cm}$, thereby achieving an antistatic effect.

\section{Introduction}

Because of its excellent properties, epoxy resin has very important roles in aerospace, electronics, electrical appliances, and building materials. However, it is insulated, so the surface can easily accumulate static charge. This generated static charge does serious harm to industrial production and personal safety, and even cause explosions. Therefore, anti-static modification of epoxy resin is becoming a significant research direction.

The main methods of antistatic modification for polymer materials are formation of an electronically conductive layer on the surface of a matrix (which is easily damaged or peeled off under external action, thereby affecting the stability of electrical conductivity) and addition of electrically conductive materials into the polymer matrix to form an "antistatic network". ${ }^{1,2}$ The antistatic properties of the materials prepared by the latter method are stable, but the structure of the antistatic network is difficult to control.

In the present study, an antistatic epoxy composite with multi-walled carbon nanotube (MWCNT)-functionalized melamine foam as antistatic structure was synthesized. First, preembedded antistatic melamine foam was prepared, which was then used to prepare an antistatic epoxy composite. Melamine foam as a new type of polymer foam has received extensive

Key Laboratory of Carbon Fiber and Functional Polymers (Beijing University of Chemical Technology), Ministry of Education, College of Materials Science and Engineering, Beijing University of Chemical Technology, Beijing 100029, PR China. E-mail: zouwei@mail.buct.edu.cn; Tel: +86-10-64430908 attention due to its relatively low density, ${ }^{3}$ sound absorption, ${ }^{4}$ fire retardance ${ }^{5}$ and high thermal stability. ${ }^{6,7}$ However, in the field of antistatic applications, the high expansion ratio of insulator melamine foam ${ }^{8}$ makes it difficult for an antistatic agent to form an antistatic network at low filling content, which is a high cost factor. As permanent antistatic fillers, MWCNTs are good choices for use in a melamine resin matrix due to their high aspect ratio. ${ }^{9-11}$ However, because of the inertia of MWCNT surfaces, MWCNTs agglomerate readily, which affects their uniform dispersion in the polymer matrix. ${ }^{12,13}$ Therefore, solving the problem of the dispersion of MWCNTs in polymer nanocomposites is important.

The most promising method to modify the surface of MWCNTs is surface chemical functionalization. ${ }^{14,15}$ After an agent with a similar polar or chemical structure to the target matrix is grafted onto MWCNTs, a compatible interface which could graft to another organism or improve the dispersion of MWCNTs in the nanocomposite is formed, which will enable the antistatic polymer nanocomposite to achieve percolation with lower MWCNT content. Traditionally, carbon nanotubes are modified with acids, but this leads to a reduction of the length of MWCNTs and more structural defects in the acidified MWCNTs. ${ }^{16,17}$ However, a method for direct functional modification of the sidewalls of carbon nanotubes using compounds with excess electrons, such as nitrene and carbene, can not only avoid carbon nanotubes from being cut off when carbon nanotubes are subjected to strong-acid treatment, but also simplify the step of treating the surface of the MWCNTs. ${ }^{18,19}$ Therefore, this method has garnered increasing attention. In 
our work, MWCNTs were modified using this method. Thermal decomposition of azide groups can produce nitrene structures, so azide polyacrylic acid was selected as the surfacemodification agent of MWCNTs. Then, melamine was covalently bonded to the MWCNTs using the carboxyl groups in azide polyacrylic acid. Subsequently, paraformaldehyde and melamine were added to the reaction system to react, and the melamine-formaldehyde prepolymer was obtained. After the foaming agent and curing agent were added and mixed homogeneously, the MWCNTs/melamine foam nanocomposite was obtained by microwave foaming. The antistatic melamine foam nanocomposite percolated if the filling amount of MWCNTs was $2.4 \mathrm{~kg} \mathrm{~m}^{-3}$. Finally, the prepared antistatic foam at different compression ratios was immersed in epoxy resin before the latter was cured. When the compression ratio reached $40 \%$, the antistatic effect was achieved.

\section{Experimental}

\subsection{Materials}

MWCNTs (purity $\geq 95 \%$; diameter $10-20 \mathrm{~nm}$; length $0.5-500$ $\mu \mathrm{m})$ were provided from Shenzhen Nanotech. Sodium azide was purchased from Qixia Chemicals. Acryloyl chloride was obtained from Energy and Chemical Industry. Acetone (99.5\%), ethanol (99.7\%) and 1,4-dioxane were purchased from Beijing Chemical Factory. Sodium dodecyl benzene sulfonate (SDBS, 99\%) was obtained from Sinopharm Chemical Reagents. Azobisisobutyronitrile (AIBN) was provided by Beijing Chemical Reagents. Paraformaldehyde (POM, 95\%), melamine, $n$-pentane (99\%), N,N-dimethylacetamide (DMAC, 99.8\%), sodium polyphosphate (99\%), emulsifier OP-10, monoethanolamine (MEA) and sodium formate (99.5\%) were purchased from $\mathrm{Fu}$ Chen Chemical Reagent Factory. Anhydrous formic acid was provided by Tianjin Kwangfu Fine Chemical Industry Research Institute. Diglycidyl ether of bisphenol A (DGEBA) was purchased from Heli Resins with the epoxide equivalent weight of $196 \mathrm{~g}$ per equiv. epoxy. 1,6-Diaminohexane (DAH) was obtained from Shanghai Reagents and purified by distillation under reduced pressure prior to use.

\subsection{Synthesize polyacryloyl chloride}

Polyacryloyl chloride was prepared by free-radical polymerization. First, $50 \mathrm{mg}$ of AIBN was added to 1,4-dioxane and the solution magnetically stirred at room temperature for $10 \mathrm{~min}$. The 1,4-dioxane solution of AIBN prepared above and acryloyl

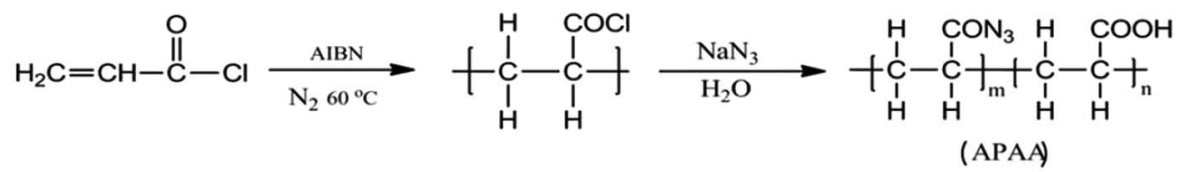

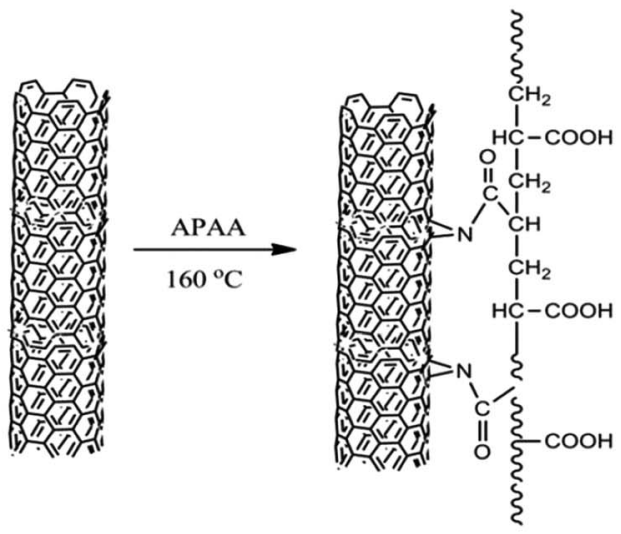

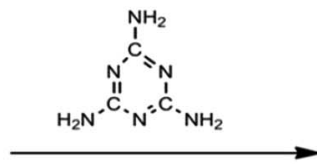

DMSO 24h

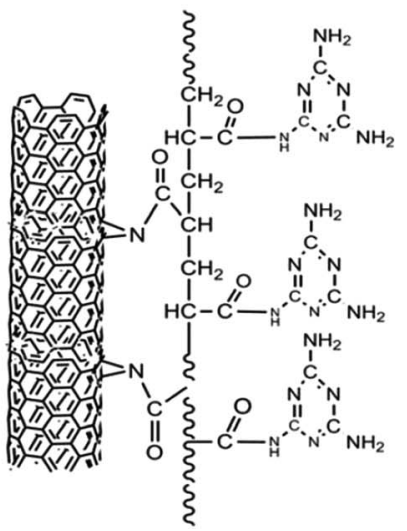

Fig. 1 Production of MA-APAA-MWCNTs (schematic). 
chloride were then injected separately into a vacuum polymerization bottle, and a mixed homogeneous solution was obtained by magnetic stirring at room temperature for $10 \mathrm{~min}$. Then, the solution was allowed to react at $60{ }^{\circ} \mathrm{C}$ for $24 \mathrm{~h}$. The product was collected and dried under vacuum. ${ }^{20}$

\subsection{Synthesis of APAA}

Polyacryloyl chloride $(1 \mathrm{~g})$ was dissolved in acetone $(10 \mathrm{ml})$ and magnetically stirred in an ice bath. During stirring, $10 \%$ sodium azide solution $(8 \mathrm{ml})$ was prepared and added dropwise gradually to an acetone solution of polyacryloyl chloride. After $4 \mathrm{~h}$, APAA was obtained.

\subsection{Synthesis of APAA-MWCNTs}

First, $1 \mathrm{~g}$ of APAA was dissolved in $10 \mathrm{ml}$ of DMAC. Then, $100 \mathrm{mg}$ of MWCNTs were dispersed homogeneously in $50 \mathrm{ml}$ of NDMAC. Then, a DMAC solution of APAA was added dropwise to the MWCNTs suspension and stirred for $2 \mathrm{~h}$ at $160{ }^{\circ} \mathrm{C}$. Finally, the resulting sample was respectively washed thrice with acetone and deionized water and dried under vacuum. ${ }^{21}$

\subsection{Synthesis of MA-APAA-MWCNTs}

APAA-MWCNTs $(1 \mathrm{~g})$ were dispersed in DMAC $(10 \mathrm{ml})$ and underwent ultrasound for $30 \mathrm{~min}$. Melamine $(870 \mathrm{mg}$ ) was dissolved in dimethyl sulfoxide $(50 \mathrm{ml})$ and the solution obtained was then added into the APAA-MWCNTs suspension in which $0.5 \% \quad N, N^{\prime}$-dicyclohexylcarbodiimide (DCC) was dissolved as a condensation agent. After the reaction had been carried out at $160{ }^{\circ} \mathrm{C}$ for $6 \mathrm{~h}$, the product was obtained and cleaned repeatedly with dimethyl sulfoxide and acetone, and dried under vacuum. A schematic diagram of MA-APAAMWCNTs is shown in Fig. 1.

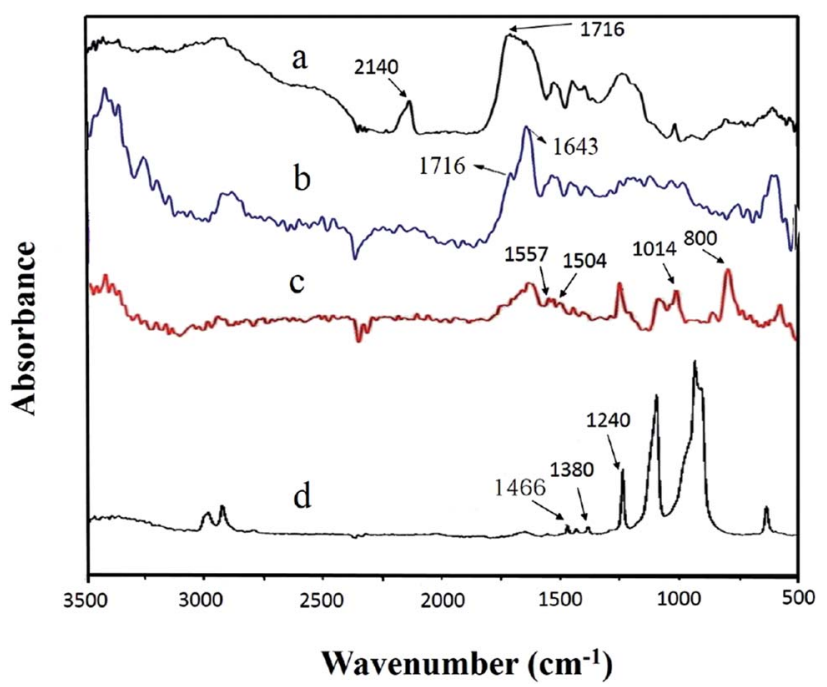

Fig. 2 FT-IR spectra of azide polyacrylic acid (a), APAA-MWCNTs (b), MA-APAA-MWCNTs (c) and MA-APAA-MWCNTs/POM (d).

\subsection{Synthesis of melamine foam/MA-APAA-MWCNTs nanocomposite}

First, $7.5 \mathrm{~g}$ of water, $0.3 \mathrm{~g}$ of sodium formate, $0.099 \mathrm{~g}$ of sodium polyphosphate and a certain amount of MA-APAA-MWCNTs were mixed and dispersed by ultrasound for $30 \mathrm{~min}$. Then, $9.7 \mathrm{~g}$ of POM was added to the mixture and stirred at $75^{\circ} \mathrm{C}$ for $10 \mathrm{~min}$. After that, $12.76 \mathrm{~g}$ of melamine was added at $75^{\circ} \mathrm{C}$ and stirred for $40 \mathrm{~min}$, and a prepolymer was obtained. After the prepolymer suspension was ice-cooled rapidly, the emulsifier, $0.15 \mathrm{~g}$ of OP10, $1.05 \mathrm{~g}$ of SDBS and $6 \mathrm{~g}$ of pentane (blowing agent) were introduced into the prepolymer suspension and stirred at high speed. Then, $1.2 \mathrm{~g}$ of formic acid was added. A melamine foam nanocomposite was obtained by microwavefoaming.

\subsection{Synthesis of epoxy resin/melamine foam/MA-APAA- MWCNTs composite}

DGEBA and DAH (epoxy/N-H $=1: 1 \mathrm{~mol}$ ) were mixed thoroughly at $45^{\circ} \mathrm{C}$ to form a clear solution. The mixture was poured immediately into a mold made by the polypropylene-containing melamine foam/MA-APAA-MWCNTs nanocomposite, which was at a certain compression ratio. The mold was then transferred to the oven under reduced pressure for $5 \mathrm{~min}$ to remove entrapped air bubbles. Finally, the epoxy resin was cured in an air-blast oven at a schedule of $70{ }^{\circ} \mathrm{C} / 1.5 \mathrm{~h}+150{ }^{\circ} \mathrm{C} / 2.5 \mathrm{~h} .{ }^{22}$ Subsequently, the epoxy resin/melamine foam/MA-APAAMWCNTs composite was prepared.

\subsection{Characterization}

Raman spectroscopy (wavelength $532 \mathrm{~nm}$ ) using a HR800 system (JY) was used to test the surface structure of MWCNTs. Fourier-transform infrared spectrometry (FT-IR; 670; NicoletNexus) was carried out using pellet samples with $\mathrm{KBr}$. Energy dispersive spectrometry (EDS) was conducted using a scanning electron microscope. (S4700; Hitachi). Differential scanning calorimetry (DSC) was done to understand the thermal behavior between MWCNTs and POM (the standard samples used in DSC were indium and zinc, and the uncertainties of the temperature and $\Delta H$ were, respectively, $\pm 0.5{ }^{\circ} \mathrm{C}$ and $\left.\pm 0.1 \mathrm{~J} \mathrm{~g}^{-1}\right)$. Highresolution transmission electron microscopy (HR-TEM) using an electron microscope (JEM-3010; JEOL) and scanning electron microscopy (SEM) using a S4700 system (Hitachi) were used to observe sample morphology. Surface resistivity was measured by a four-point probe method using a four-probe meter (ST2258A; Suzhou Jingge Electronics). Measurements of volume resistivity were taken using a digital high resistance meter (6517A; Keithley).

\section{Results and discussion}

\subsection{Preparation of APAA-MWCNTS}

Infrared spectroscopy is an effective method to characterize a change of functional groups before or after MWCNTs grafting. The FT-IR spectrum for azide polyacrylic acid is shown in Fig. 2a; there were obvious absorption peaks at wavenumbers of $1716 \mathrm{~cm}^{-1}$ and $2140 \mathrm{~cm}^{-1}$, which is the characteristic stretching 


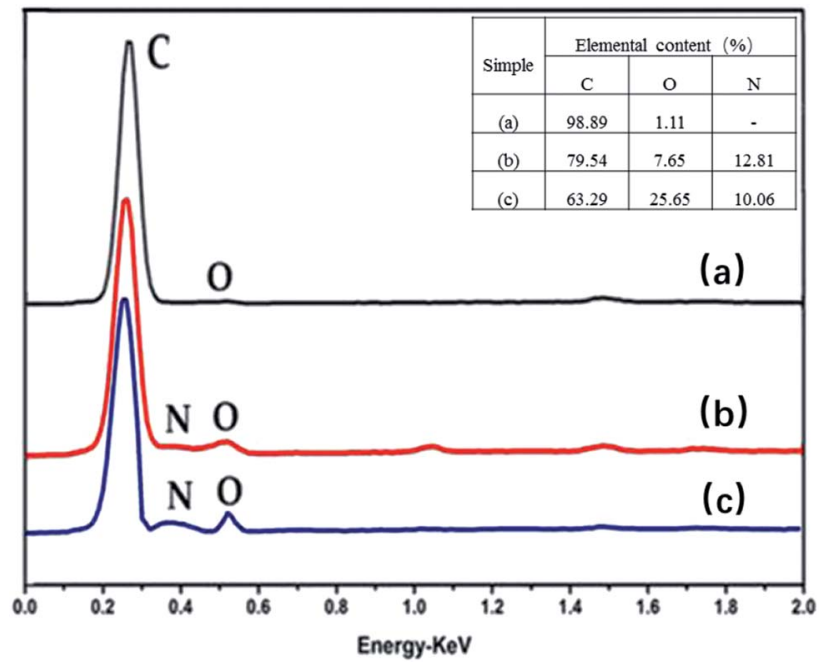

Fig. 3 EDS spectra of MWCNTs (a), APAA-MWCNTs (b) and MA-APAAMWCNTS (c).

vibration peak of the carboxyl group and azide bond $\left(-\mathrm{N}_{3}\right)$, respectively. When MWCNTs were functionalized with azide polyacrylic acid, as shown in Fig. $2 \mathrm{~b}, \mathrm{~N}-\mathrm{C}\left(1643 \mathrm{~cm}^{-1}\right)$ and $\mathrm{C}=\mathrm{O}$ $\left(1716 \mathrm{~cm}^{-1}\right)$ stretching vibrations of an amide were observed. The EDS spectra are shown in Fig. 3. After azide polyacrylic acid had been grafted onto MWCNTs, the EDS spectrum (Fig. 3b) showed that the nitrogen element at $0.39 \mathrm{keV}$ was present and its content was $7.65 \mathrm{wt} \%$. The oxygen element content at 0.53 $\mathrm{keV}$ was improved, which was caused by the carboxyl groups of the azide polyacrylic acid. TGA revealed that pristine MWCNTs had no weight loss until $800^{\circ} \mathrm{C}$ in nitrogen. However, the weight of APAA-MWCNTs (Fig. 6b) remained at $55 \mathrm{wt} \%$ at $800{ }^{\circ} \mathrm{C}$. The $45 \mathrm{wt} \%$ weight loss arose from azidized polyacrylic acid being grafted onto MWCNTs.

Fig. 4 shows the surface morphology of pristine MWCNTs (a) and APAA-MWCNTs (b), respectively. Compared with the original MWCNTs, the surface of MWNCTs grafted with azide polyacrylic acid was wrapped around a layer of material; this was evidence that azidized polyacrylic acid had been grafted onto MWCNTs.

To verify the low loss of modified MWCNTs (the side walls of which were directly covalently functionalized by azidized

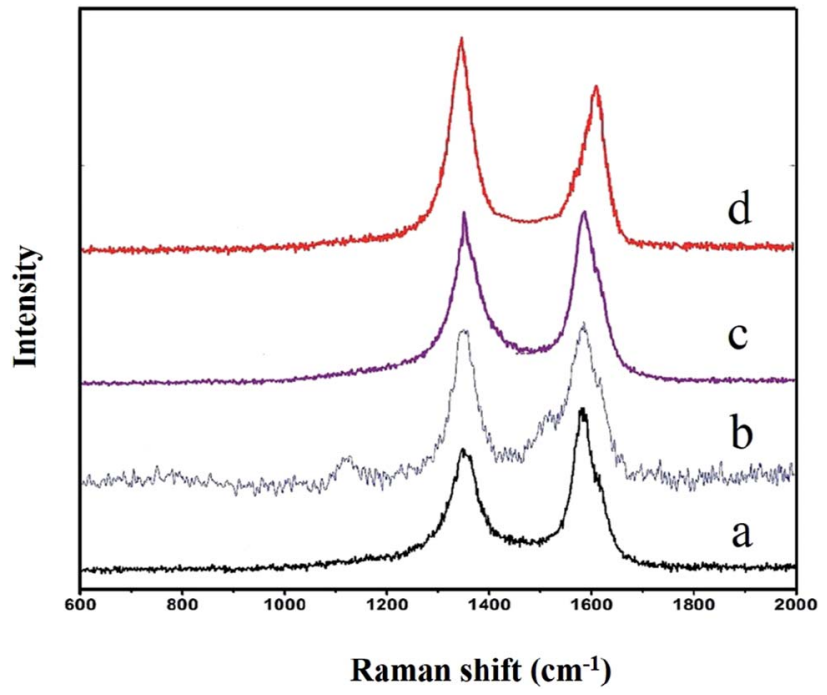

Fig. 5 Raman spectra of pristine MWCNTs (a), APAA-MWCNTs (b), acid-treated MWCNTs (c) and MA-APAA-MWCNTs (d).

polyacrylic acid), Raman spectroscopy of pristine MWCNTs (a), APAA-MWCNTs (b) and acid-treated MWCNTs (c) are presented in Fig. 5 . The intensity ratio of the D over the G peak $\left(I_{\mathrm{D}} / I_{\mathrm{G}}\right)$ of the original MWCNTs was 0.85. After the original MWCNTs had been modified by azide polyacrylic acid, the $I_{\mathrm{D}} / I_{\mathrm{G}}$ of the APAAMWCNTs increased to 0.91 , which was due to the cyclicaddition reaction of the MWCNTs and the nitrile structure. In addition, the $I_{\mathrm{D}} / I_{\mathrm{G}}$ of the acidified MWCNTs was higher than that of the original MWCNTs and APAA-MWCNTs. This was because, after oxidation of the mixed acid, the carbon structure of the MWCNTs changed so that the surface of MWCNTs had multiple defects. Hence, the MWCNTs covalently functionalized by azide polyacrylic acid were less damaged.

\subsection{Preparation of MA-APAA-MWCNTs}

Fig. 2c shows the infrared images of MA-APAA-MWCNTs; wavenumbers $\left(\mathrm{cm}^{-1}\right)$ at $800,1014,1504$ and 1557 represent, respectively, deformation, vibration of the triazine ring derived from melamine, torsional vibration of the $\mathrm{N}-\mathrm{H}$ bond, and the stretching vibration and skeletal vibration of the $\mathrm{C}=\mathrm{N}$ bond. In the EDS spectra, the curve of MA-APAA-MWCNTs (Fig. 3c)

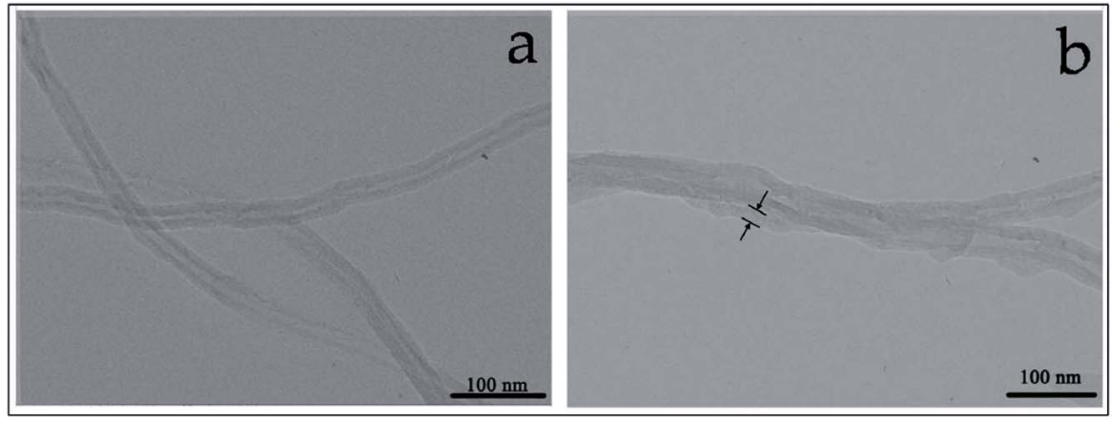

Fig. 4 TEM images of pristine MWCNTs (a) and APAA-MWCNTs (b). 


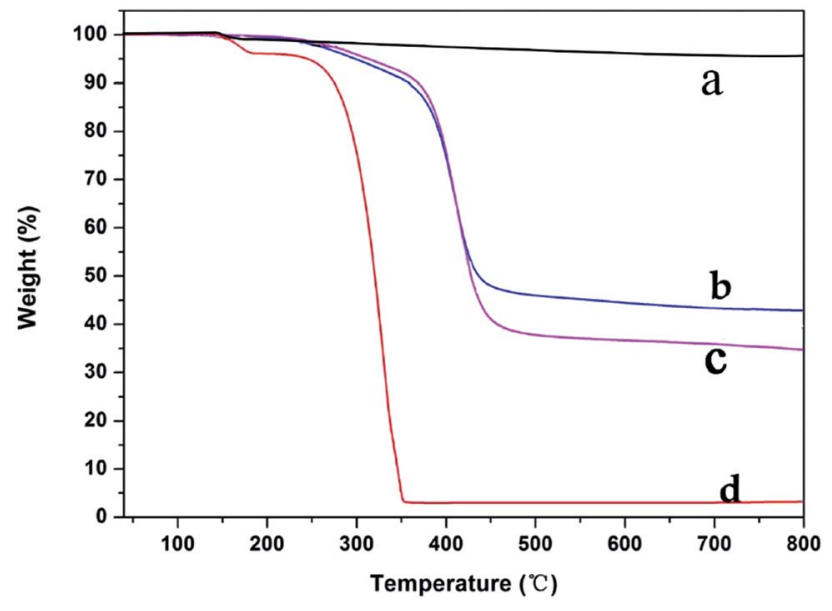

Fig. 6 TGA of pristine MWCNTs (a), APAA-MWCNTs (b), MA-APAAMWCNTs (c) and melamine (d).

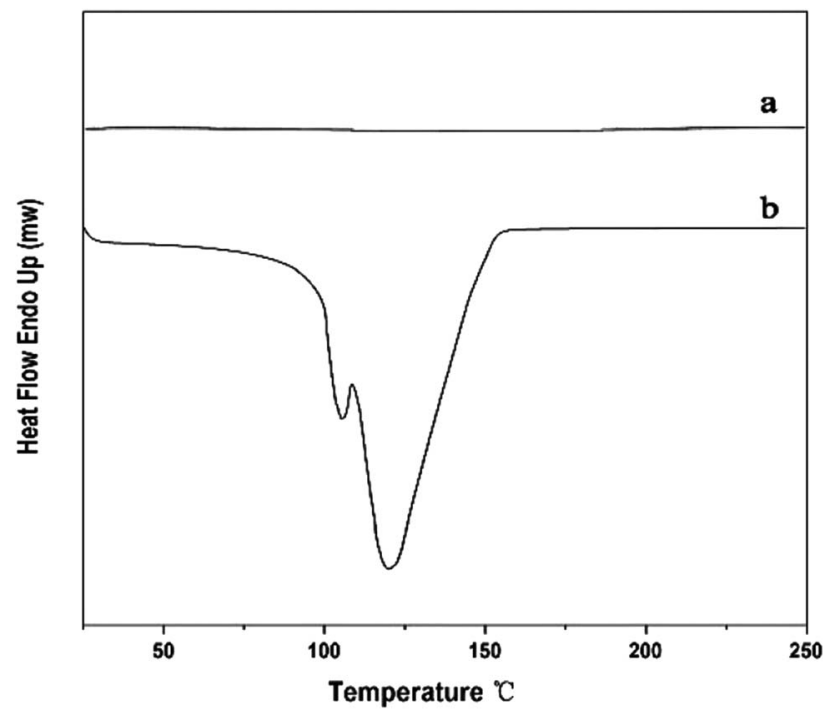

Fig. 7 DSC curve of pristine MWCNTs/POM (a) and MA-APAAMWCNTS/POM mixtures (b).

showed the nitrogen element content increased more than twofold, which was mainly because the melamine (in which the content of nitrogen element was $\approx 72.7 \mathrm{wt} \%$ ) was grafted onto MWCNTs. In Raman spectra, the G-band of MA-APAA-MWCNTs (Fig. 5d) had a blue shift relative to that of APAA-MWCNTs (Fig. 5c), which was evidence that melamine had been grafted onto MWCNTs.

TGA curves could be used to estimate the weight ratio of the grafted organic material on MWCNTs. The weight of APAAMWCNTs (Fig. 6b), MA-APAA-MWCNTs (Fig. 6c) and melamine (Fig. 6d), respectively, remained at $45 \mathrm{wt} \%, 36 \mathrm{wt} \%$ and $3 \mathrm{wt} \%$ at $800{ }^{\circ} \mathrm{C}$. The weight fraction of melamine grafted onto the MWCNTs in the nanocomposite could be estimated using the equation:

$$
B \%=(1-X) \times A \%+X \times C \%
$$

where $A \%, B \%$, and $C \%$ were the weight loss percent of APAAMWCNTs, MA-APAA-MWCNTs, and melamine at $800{ }^{\circ} \mathrm{C}$, respectively, and $\mathrm{X}$ denotes the weight fraction of melamine grafted onto MWCNTs. The amount of melamine grafted was $\approx 21 \mathrm{wt} \%$.

\subsection{Properties of melamine foam/MA-APAA-MWCNTs nanocomposite}

Fig. 7 shows the DSC curve of pristine MWCNTs/POM and MAAPAA-MWCNTS/POM mixtures. Compared with pristine MWCNTs/POM, MA-APAA-MWCNTs mixed with POM had two obvious exothermic peaks at around $104{ }^{\circ} \mathrm{C}$ and $117^{\circ} \mathrm{C}$, which represented the addition reaction of melamine grafted onto MWCNTs and the polycondensation reaction between formaldehyde and methylol melamine. Through exothermic reactions, the MWCNTs and foam matrix formed a good chemical-bond interface. To further demonstrate the above reaction, the reaction mixture was texted by FT-IR. The stretching vibration peaks for $\mathrm{C}-\mathrm{O}-\mathrm{C}\left(1240 \mathrm{~cm}^{-1}\right)$, primary alcohols $\left(1380 \mathrm{~cm}^{-1}\right)$ and $-\mathrm{CH}_{2}-\left(1460 \mathrm{~cm}^{-1}\right)$ were observed (Fig. $\left.2 \mathrm{~d}\right)$. This suggested that MA-APAA-MWCNTs were involved in the formation of melamine resin by the reaction with POM.

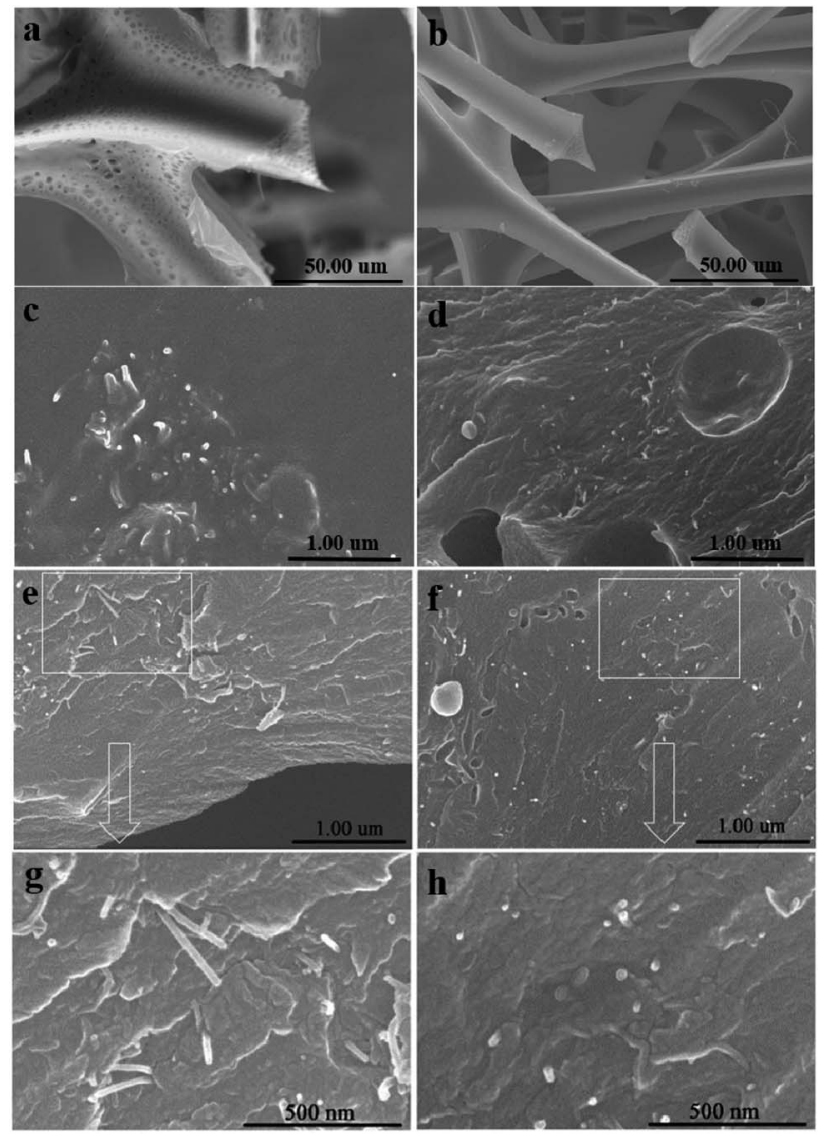

Fig. 8 The skeleton of melamine foam without MWCNTs (a) and melamine foam/MA-APAA-MWCNTs with loading of MWCNTs as 2.4 $\mathrm{kg} \mathrm{m}^{-3}(\mathrm{~b})$; the fracture surface of melamine foam/pristine MWCNTs with loading of MWCNTs as 1.1 and $2.4 \mathrm{~kg} \mathrm{~m}^{-3}$ ( $\mathrm{c}$ and e) and melamine foam/MA-APAA-MWCNTs with loading of MWCNTs as 1.1 and $2.4 \mathrm{~kg}$ $\mathrm{m}^{-3}$ ( $d$ and $\mathrm{f}$ ); partial higher magnification images of (e) and (f) ( $\mathrm{g}$ and $\mathrm{h}$ ). 


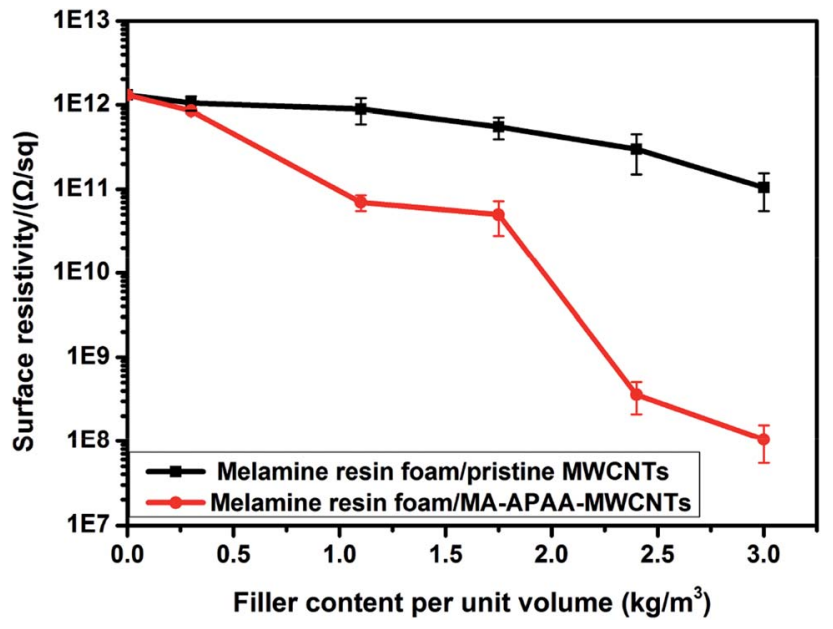

Fig. 9 Surface resistivity of melamine foam/pristine MWCNTs (a) and melamine foam/MA-APAA-MWCNTs (b) nanocomposite.

The skeleton of melamine foam without MWCNTs and melamine foam/MA-APAA-MWCNTs and the fracture surfaces of melamine foam/pristine MWCNTs and melamine foam/MAAPAA-MWCNTs were investigated by SEM (Fig. 8). As shown in Fig. 8b, compared with the skeleton of melamine foam without addition of MWCNTs (Fig. 8a), defects on the skeleton of melamine foam/MA-APAA-MWCNTs were greatly reduced. In Fig. $8 \mathrm{c}$ and e, the pristine MWCNTs in the melamine foam matrix are not uniformly dispersed and agglomerate, whereas the MA-APAA-MWCNTs have good dispersion (Fig. 8d and f). In addition, more details could be observed from highermagnification images. At the fracture surface of the melamine foam/pristine MWCNTs composite (Fig. 8g), most of the MWCNTs were "pulled" out in the cell walls, which was caused by the interface interaction between MWCNTs and the matrix being poor. However, the MA-APAA-MWCNTs were found to be broken, embedded and tightly held to the matrix (Fig. 8h). It was reasonable to deduce that after MWCNTs had been grafted with melamine, MWCNTs utilized the surface functional groups to participate in polymerization, which improved the affinity between MWCNTs and the matrix.
In Fig. 9, the surface resistivity decreased steadily with increasing loadings of pristine MWCNTs and MA-APAAMWCNTs. In particular, because MWCNTs grafted with melamine and melamine resin have good compatibility, MA-APAAMWCNTs reduced surface resistivity considerably. When the filling amount of MWCNTs was $2.4 \mathrm{~kg} \mathrm{~m}^{-3}$, melamine foam/ MA-APAA-MWCNTs appeared to percolate to achieve an antistatic effect. In addition, the density of the antistatic melamine foam synthesized above was only $10.4 \mathrm{~kg} \mathrm{~m}^{-3}$.

\subsection{Properties of epoxy resin/melamine foam/MA-APAA- MWCNTs composite}

The melamine foam/MA-APAA-MWCNTs nanocomposite, whose filling amount of MWCNTs was $2.4 \mathrm{~kg} \mathrm{~m}^{-3}$, was used to explore the relationship between the surface resistivity and volume resistivity of epoxy resin/melamine foam/MA-APAAMWCNTs composite and compression ratio of melamine foam/MA-APAA-MWCNTs nanocomposite. Fig. 10 shows that the surface resistivity and volume resistivity of the prepared epoxy composite decreased gradually as the compression ratio of the antistatic foam increased. When the compression ratio reached $40 \%$, the epoxy resin/melamine foam/MA-APAAMWCNTs composite appeared to percolate and the surface resistivity and volume resistivity, respectively, reached $1.05 \times$ $10^{8} \Omega \mathrm{sq}^{-1}$ and $3.5 \times 10^{8} \Omega \mathrm{cm}$, so an antistatic effect was achieved.

\section{Conclusions}

We described a new method of preparing an antistatic epoxy composite. A method for direct functional modification of the sidewalls of MWCNTs using azide polyacrylic acid was utilized to avoid MWCNTs from being cut off, and melamine was then covalently bonded to the MWCNTs. The MWCNTs utilized surface functional groups to participate in the preparation of melamine foam, which improved the compatibility of the MWCNTs with the matrix. As a result, the surface resistivity of melamine foam matrix nanocomposite reached $3.6 \times 10^{8} \Omega$ $\mathrm{sq}^{-1}$ when the loading of MWCNTs was $2.4 \mathrm{~kg} \mathrm{~m}^{-3}$. After that, the antistatic foam, the density of which was only $10.4 \mathrm{~kg} \mathrm{~m}^{-3}$,
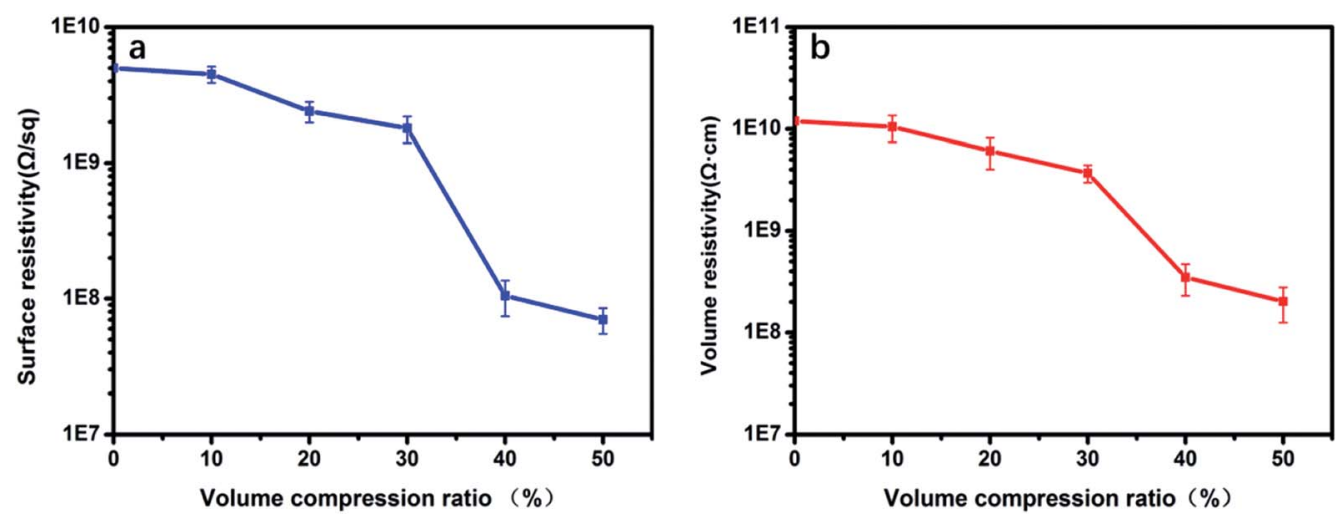

Fig. 10 Relationship between the surface resistivity (a), volume resistivity (b) of epoxy resin/melamine foam/MA-APAA-MWCNTs composite and compression ratio of melamine foam/MA-APAA-MWCNTs nanocomposite. 
was immersed in the epoxy resin before the epoxy resin was cured. An epoxy composite having antistatic properties was obtained by controlling the compression ratio of the antistatic foam during curing. When the compression ratio reached $40 \%$, the surface resistivity and volume resistivity, respectively, reached $1.05 \times 10^{8} \Omega \mathrm{sq}^{-1}$ and $3.5 \times 10^{8} \Omega \mathrm{cm}$, which indicated that an antistatic effect had been achieved.

\section{Conflicts of interest}

There are no conflicts to declare.

\section{Acknowledgements}

This work was supported by the National Natural Science Foundation of China (51203007).

\section{References}

1 Y. Xu, Y. Li, W. Xu and J. Bao, J. Mater. Sci.: Mater. Electron., 2015, 26, 1159-1171.

2 Y. Zhao, W. Wu, J. Chen, H. Zou, L. Hu and G. Chu, Ind. Eng. Chem. Res., 2013, 51, 3811-3818.

3 R. Schlitt, F. Bodendieck and F. Serène, J. Chromatogr. A, 2001, 467, 121-128.

4 J. P. Arenas and M. J. Crocker, Sound. Vib., 2010, 44, 12-18. 5 S. Hörold, Polym. Degrad. Stab., 1999, 64, 427-431.

6 C. A. Lukey, D. J. T. Hill and P. J. Pomery, Polym. Degrad. Stab., 2002, 78, 485-490.

7 C. Devallencourt, J. M. Saiter, A. Fafet and E. Ubrich, Thermochim. Acta, 1995, 259, 143-151.

8 M. Pruneanu, S. S. Maier, O. Niculescu, F. Vitan and V. Deselnicu, Rev. Chim., 2011, 62, 60-63.
9 J. Guo, Y. Liu, R. Prada-Silvy, Y. Tan, S. Azad, B. Krause, P. Pötschke and B. P. Grady, J. Polym. Sci., Part B: Polym. Phys., 2014, 52, 73-83.

10 Y. J. Kim, T. S. Shin, H. D. Choi, J. H. Kwon, Y. C. Chung and H. G. Yoo, Carbon, 2005, 43, 23-30.

11 Z. Spitalsky, D. Tasis, K. Papagelis and C. Galiotis, Prog. Polym. Sci., 2010, 35, 357-401.

12 Y. Li, R. Zhang, T. Wang, Y. Wang, Y. Wang, L. Li, W. Zhao, X. Wang and J. Luo, Talanta, 2016, 154, 99-108.

13 S. Chen, Z. Wei, L. Guo, W. Ding, L. Dong, P. Shen, X. Qi and L. Li, Chem. Commun., 2011, 47, 10984-10986.

14 F. H. Gojny, J. Nastalczyk, Z. Roslaniec and K. Schulte, Chem. Phys. Lett., 2003, 370, 820-824.

15 M. Abdalla, D. Dean, P. Robinson and E. Nyairo, Polymer, 2008, 49, 3310-3317.

16 X. Li, H. Zhou, C. Fu, F. Wang, Y. Ding and Y. Kuang, Sens. Actuators, B, 2016, 236, 144-152.

17 R. Yang, Y. Zheng, P. Li, H. Bai, Y. Wang and L. Chen, RSC Adv., 2016, 6, 85970-85977.

18 C. Gao, H. He, L. Zhou, X. Zheng and Y. Zhang, Chem. Mater., 2008, 21, 360-370.

19 E. J. Lawrence, G. G. Wildgoose, L. Aldous, Y. A. Wu, J. H. Warner, R. G. Compton and P. D. Mcnaughter, Chem. Mater., 2011, 23, 3740-3751.

20 D. Sinirlioglu, A. Aslan, A. E. Muftuoglu and A. Bozkurt, J. Appl. Polym. Sci., 2014, 131, 1001-1007.

21 X. Wang, C. Zhang, Z. Du, H. Li and W. Zou, Polym. Adv. Technol., 2016, 28, 791-796.

22 J. Wan, C. Li, H. Fan and B. G. Li, Ind. Eng. Chem. Res., 2017, 56, 4938-4948. 